Spin pumping and inverse spin Hall effect in antiferromagnetic exchange coupled [Co/ $\mathrm{Ru} / \mathrm{Co}$ ]/Pt heterostructures

L. Avilés-Félix, A. Butera, and J. E. Gómez

Citation: Appl. Phys. Lett. 110, 052402 (2017); doi: 10.1063/1.4975375

View online: http://dx.doi.org/10.1063/1.4975375

View Table of Contents: http://aip.scitation.org/toc/apl/110/5

Published by the American Institute of Physics

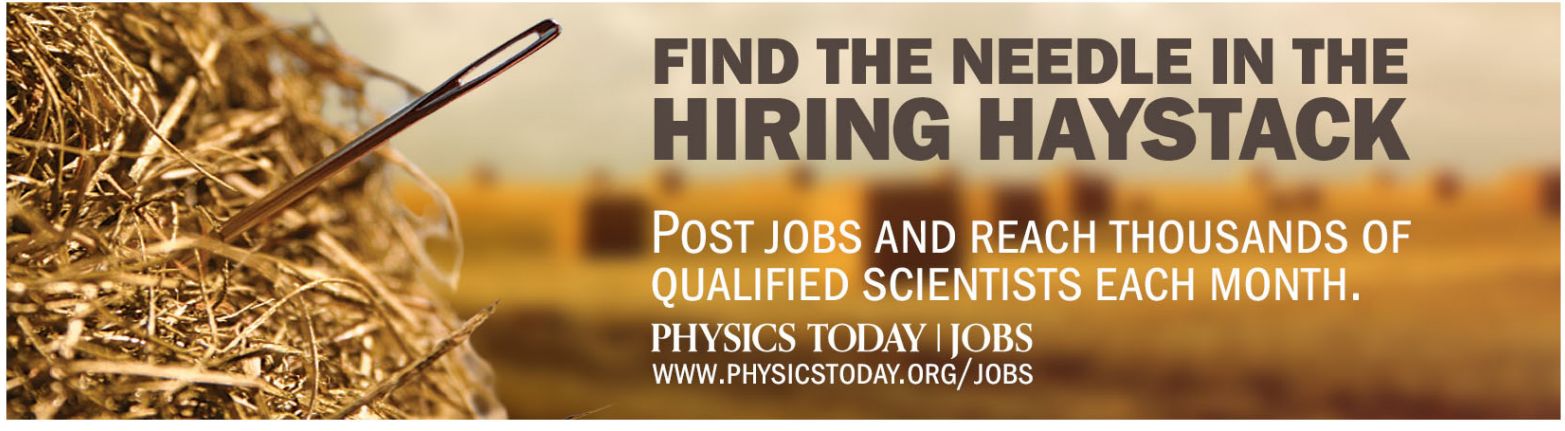




\title{
Spin pumping and inverse spin Hall effect in antiferromagnetic exchange coupled $[\mathrm{Co} / \mathrm{Ru} / \mathrm{Co}] / \mathrm{Pt}$ heterostructures
}

\author{
L. Avilés-Félix, ${ }^{\text {a),b) }}$ A. Butera, ${ }^{\text {b) }}$ and J. E. Gómez \\ Centro Atómico Bariloche, Instituto de Nanociencia y Nanotecnología (CNEA) and Conicet, 8400 Bariloche, \\ Río Negro, Argentina
}

(Received 9 December 2016; accepted 20 January 2017; published online 31 January 2017)

\begin{abstract}
We report the observation of spin pumping and inverse spin Hall effects in antiferromagnetically coupled $[\mathrm{Co} / \mathrm{Ru} / \mathrm{Co}] / \mathrm{Pt}$ heterostructures. The "spin-flop" magnetization process observed in antiferromagnetically exchange coupled Co layers combined with spin pumping and inverse spin Hall effects allowed us to detect both transversal and longitudinal charge accumulations. By controlling the exchange coupling strength and the spin flop transition in the magnetization process, it was possible to produce spin currents polarized in different directions. Published by AIP Publishing.

[http://dx.doi.org/10.1063/1.4975375]
\end{abstract}

The generation and detection of pure spin currents via spin pumping (SP) and the inverse spin Hall effect (ISHE) opened new paths in the development of spintronic devices. In recent years, these effects have been adopted and adapted for the fabrication of hybrid spintronic structures, such as organic semiconductors, ${ }^{1}$ spin valves, ${ }^{2}$ or ferromagnetic oxides-based structures. ${ }^{3}$ The recent work has proved that magnetoelectric structures combined with strong spin-orbit coupling metals can also be used to modify the magnetic field position at which the spin pumping phenomenon occurs. ${ }^{4}$ Spin pumping, known as one of the responsible phenomena for the generation and injection of a pure spin current into a metallic layer, and the inverse spin Hall effect, understood as the quantum effect that converts a spin current into a dc charge current, are two mechanisms for the generation and detection of spin currents. These effects, combined with the theory developed to describe the underlying phenomena, were used to estimate microscopic parameters related to the transport properties of ferromagnetic/normal metal bilayer systems. For example, the spin diffusion length $\left(\lambda_{S D}\right)$, spin Hall angle $\left(\Theta_{S H}\right)$, and the spin mixing conductance $\left(g^{\uparrow \downarrow}\right)$ provided a way to compare the efficiency in the generation and detection of spin polarized currents among different $5 d$ or $4 d$ transition metal elements such as $\mathrm{Pt},{ }^{5,6} \mathrm{Pd}{ }^{7}$ or $\mathrm{Ta}^{8,9}$ These studies are very important for the choice of suitable components for technological applications. The strong spin-orbit coupling in these transition metals is responsible for the ISHE, which converts a pure spin current into a transversal charge current according to $\mathbf{J}_{\mathrm{c}}=\Theta_{S H}$ $\left(\frac{-2 e}{\hbar}\right) \mathbf{J}_{\mathbf{s}} \times \boldsymbol{\sigma}$, where $\mathbf{J}_{\mathrm{c}}$ and $\mathbf{J}_{\mathbf{s}}$ are the charge current and the pumped dc spin current, respectively, and $\boldsymbol{\sigma}$ is the polarization direction of the spin current. Previous works on single ferromagnetic-normal metal (FM-NM) bilayers such as $\mathrm{Fe}_{20} \mathrm{Ni}_{80} / \mathrm{Pt},{ }^{5} \mathrm{Co} / \mathrm{Pt},{ }^{6}$ or $\mathrm{Fe}_{20} \mathrm{Ni}_{80} / \mathrm{Ru}^{8}$ were important contributions to the understanding of spin polarized currents. However, the generation of pure spin currents in new systems, easily adaptable to spintronics (as organic semiconductors or

\footnotetext{
a) Author to whom correspondence should be addressed. Electronic mail: lavilesf@gmail.com.

b) Also at Instituto Balseiro - Univ. Nac. de Cuyo, Av. Bustillo 9500, 8400 Bariloche, Rio Negro, Argentina.
}

spin valves), could lead to improvements in the performance of existing and future electronic devices. An alternative for the development of spintronic systems with new functionalities involves the incorporation of antiferromagnetic (AF) compounds in ferromagnet-normal metal bilayers for the generation or detection of spin currents. Some authors reported an enhancement of the spin to charge current conversion by using AF layers as spin-current detectors ${ }^{10}$ or as interlayers between the ferromagnet and the metallic layers. ${ }^{11,12}$

In this letter, we report the observation of SP and ISHE in a system consisting of an exchange coupled AF structure and a normal metal layer (NM) with strong spin-orbit coupling. We propose a $\mathrm{Co} / \mathrm{Ru} / \mathrm{Co}$ trilayer structure, known as a synthetic antiferromagnet (SAF), as a spin current injector into a $\mathrm{Pt}$ film. Early experiments in $\mathrm{Co} / \mathrm{Ru} / \mathrm{Co}$ trilayers revealed a Ruderman-Kittel-Kasuya-Yosida (RKKY)-like magnetic coupling between Co layers, whose sign and magnitude depends on the $\mathrm{Ru}$ thickness, $t_{\mathrm{Ru}}$. For $t_{\mathrm{Ru}}$ $\sim 0.6-0.7 \mathrm{~nm}$, a maximum in the AF coupling with an exchange field of $H_{E}$ on the order of several kOe was observed. ${ }^{13,14}$ The dynamic characterization of $\mathrm{Co} / \mathrm{Ru} / \mathrm{Co}$ trilayers studied by ferromagnetic resonance techniques (FMRs) revealed that the dispersion relation of the system strongly depends on the magnitude and sign of the exchange coupling constant. ${ }^{13}$

We fabricated SAF-NM heterostructures consisting of $\mathrm{Si} \| \mathrm{Ru}(2)\left|\left[\mathrm{Co}(4)\left|\mathrm{Ru}\left(t_{\mathrm{Ru}}\right)\right| \mathrm{Co}(4)\right]\right| \operatorname{Pt}(7)$ with different thicknesses of the $\mathrm{Ru}$ spacer $\left(t_{\mathrm{Ru}}=0.7,0.9\right.$, and $\left.1.2 \mathrm{~nm}\right)$. Numbers in parenthesis are the respective thicknesses expressed in $\mathrm{nm}$. Samples were grown on naturally oxidized single crystalline $\mathrm{Si}(100)$ substrates by dc magnetron sputtering (Co and $\mathrm{Ru}$ films) and rf sputtering (Pt layers). The films were deposited at room temperature using an Ar pressure of $3 \mathrm{mTorr}$ and $10 \mathrm{~W}$ of dc magnetron sputtering power for Co and Ru films, and $30 \mathrm{~W}$ of rf sputtering power in the case of $\mathrm{Pt}$ electrodes. Atomic force microscopy measurements allowed us to obtain a sputtering rate of $0.09,0.07$, and $0.2 \mathrm{~nm} / \mathrm{s}$ for $\mathrm{Co}, \mathrm{Ru}$, and $\mathrm{Pt}$, respectively. DC magnetization measurements were performed with an alternating gradient force magnetometer and a Kerr magnetometer at room temperature. FMR and ISHE measurements were made in a 

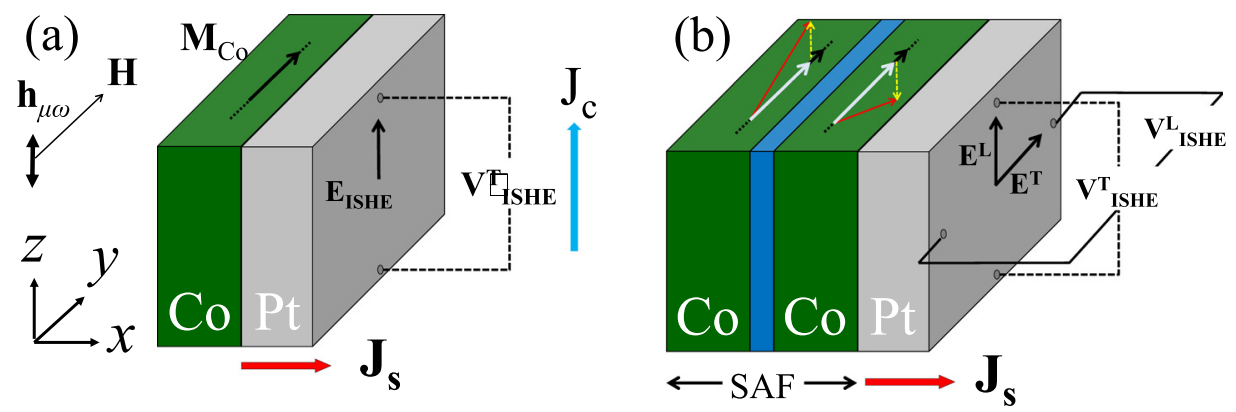

FIG. 1. (a) Schematic of the detection mechanism of a spin polarized current in a FM-NM bilayer. In resonance condition, the magnetization of the FM layer pumps a spin current $\mathbf{J}_{\mathbf{s}}$ into the NM, that is converted into a transversal charge current $\mathbf{J}_{c}$ via ISHE. (b) Schematic of the electrical contacts configuration for the detection of transversal and longitudinal ISHE voltages. The magnetization process in the SAF structure can lead to the detection of longitudinal dc voltages.

commercial Bruker ESP 300 spectrometer at a frequency of $9.40 \mathrm{GHz}$ (X-Band) using a modulation frequency of 100 $\mathrm{kHz}$ and a modulation amplitude of 10 Oe. The external magnetic field $\mathbf{H}$ and the microwave magnetic field $\mathbf{h}_{\mu \omega}$ are applied along the $y$ and $z$ axes, respectively, as sketched in Fig. 1(a). The conventional spin current detection in FM-NM bilayers is made by measuring the transversal voltage generated by ISHE on the spin current that is "pumped" into the metallic layer, as schematically shown in Fig. 1(a). It is important to mention that we are not considering the induced voltages in the underlayer of $\mathrm{Ru}(2 \mathrm{~nm})$ due to $\Theta_{S H}^{\mathrm{Ru}} \ll \Theta_{S H}^{\mathrm{Pt}} .8$ The ISHE signal was acquired by measuring the voltage between the edges of the sample, as described in previous works. ${ }^{15}$ In our case, the presence of an AF exchanged coupled structure, in which the magnetization is not necessarily aligned with the external magnetic field, opens the possibility to detect charge accumulation in unconventional directions. A schematic of the SAF-NM system and the electrical contacts configuration for the detection of SP and ISHE in the present work are shown in Fig. 1(b).

Magnetization measurements in our samples confirm that the Co layers are antiferromagnetically coupled for $t_{\mathrm{Ru}}=0.7$ and $0.9 \mathrm{~nm}$ as shown in Fig. 2(a). The magnetization process of the AF exchanged coupled Co layers consists of a spin-flop-like transition, in which the magnetizations are flipped at opposite angles from the external magnetic field and monotonically aligned with $\mathbf{H}$ until magnetic saturation is reached for $H \leqq H_{E}$. Although the individual Co layers are saturated during the magnetization process, the exchange coupling energy is not overcome until both layers are completely aligned with the external magnetic field at the exchange magnetic field value, $H_{E}$. From Fig. 2(a), we estimated the values of the $\mathrm{AF}$ exchange field as $H_{E} \sim 3020 \mathrm{Oe}$ for $t_{\mathrm{Ru}}=0.7 \mathrm{~nm}$ and $H_{E} \sim 920 \mathrm{Oe}$ for $t_{\mathrm{Ru}}=0.9 \mathrm{~nm}$. For $t_{\mathrm{Ru}}=1.2 \mathrm{~nm}$, the shape of the hysteresis loop suggests the presence of ferromagnetic coupling.

The $H_{E}$ and the saturation magnetization values $M_{s}$ extracted from the $M(H)$ curves allowed us to obtain, through numerical simulation, the FMR in-plane dispersion relations of the acoustic modes for different values of $t_{\mathrm{Ru}}$, as shown in Fig. 2(b). The model that was used model for the numerical simulation was adapted from Zhang et al. The resonance field $\left(H_{R}\right)$ values at the $\mathrm{X}$-band frequency (horizontal solid line) are indicated by filled circles in Fig. 2(b) revealing the influence of $H_{E}$ on the magnetization dynamics of the Co layers. We compared the $H_{R}$ values extracted from the inplane FMR measurements (Fig. 2(c)) with those obtained from the simulated dispersion relations plotted in Fig. 2(b). The experimental and simulated values of $H_{R}$ coincide within the error, showing an increase of $H_{R}$ when $t_{\mathrm{Ru}}$ is decreased, originated by the thickness dependence of $H_{E}$. Experimental and simulated $H_{R}$ and $H_{E}$ values as a function of $t_{\mathrm{Ru}}$ are presented in Table I. As discussed in Ref. 13, only the acoustic mode where the magnetizations of both Co layers process in phase is observed.
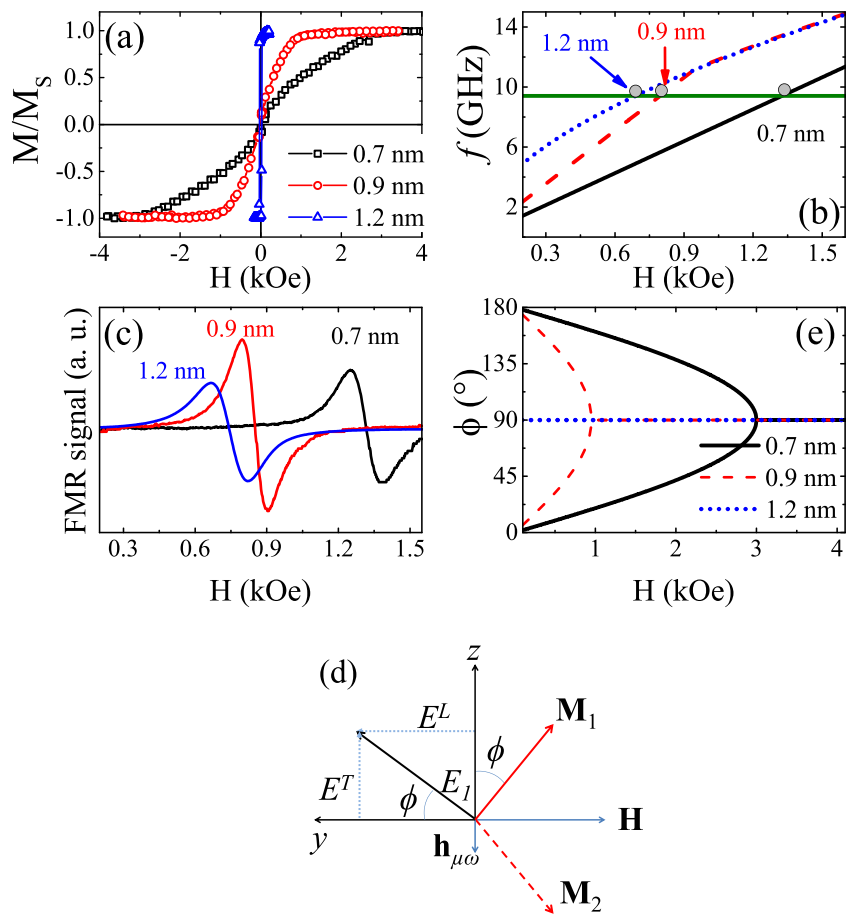

FIG. 2. (a) In plane $M(H)$ curves of $\left[\mathrm{Co} / \mathrm{Ru}\left(t_{\mathrm{Ru}}\right) / \mathrm{Co}\right] / \mathrm{Pt}$ heterostructures as a function of $t_{\mathrm{Ru}}$. (b) Simulated dispersion relations for coupled Co layers varying $t_{\mathrm{Ru}}: 0.7 \mathrm{~nm}$ (solid line), $0.9 \mathrm{~nm}$ (dashed line), and $1.2 \mathrm{~nm}$ (dotted line). The filled circles indicate the values of the resonance fields at the frequency of X-band $(9.40 \mathrm{GHz})$. An effective shape anisotropy field $4 \pi \mathrm{M}_{\text {eff }}=1.38 \times 10^{4}$ Gauss for the Co layers and a gyromagnetic factor value $g=2.14$ were used. (c) FMR absorption spectra of the SAF-NM systems varying $t_{\mathrm{Ru}}$. (d) Schematics of the misalignment of the magnetization vectors $\mathbf{M}_{1}$ and $\mathbf{M}_{2}$ of the Co layers due to the spin-flop transition. $\mathbf{M}_{1}$ corresponds to the magnetization of the Co layer closer to the Pt film. (e) Simulated magnetization equilibrium angles $\phi$ of the Co layers as a function of the external magnetic field. 
TABLE I. Resonance and exchange fields of the coupled $\mathrm{Co}(4 \mathrm{~nm}) / \mathrm{Ru}\left(t_{\mathrm{Ru}}\right) /$ $\mathrm{Co}(4 \mathrm{~nm})$ trilayers as a function of $t_{\mathrm{Ru}}$.

\begin{tabular}{lcccc}
\hline \hline$t_{\mathrm{Ru}}(\mathrm{nm})$ & $H_{E}^{\exp }(\mathrm{Oe})$ & $H_{R}^{\exp }(\mathrm{Oe})$ & $H_{E}^{\text {sim }}(\mathrm{Oe})$ & $H_{R}^{\text {sim }}(\mathrm{Oe})$ \\
\hline 0.7 & 3021 & 1322 & 3000 & 1328 \\
0.9 & 913 & 853 & 950 & 870 \\
1.2 & $\cdots$ & 680 & $\cdots$ & 740 \\
\hline \hline
\end{tabular}

We emphasize that during the spin-flop transition, the magnetization of each layer is rotated at a certain angle away from the magnetic field direction, followed by a continuous rotation towards the direction of the magnetic field. The initial rotation, described by the angle $\phi$ formed by the magnetization of the Co layers and the $z$ axis, is schematically shown in Fig. 2(d) and depends on $H$ and $H_{E}\left(\phi=90^{\circ}\right.$ for $H=H_{E}$ corresponds to $\mathbf{M}_{i} \| \mathbf{H}$ ). In our case, the magnetization equilibrium angles $(\phi)$ of the Co layers as a function of the external magnetic field were obtained from the numerical calculations and plotted in Fig. 2(e). As expected, for ferromagnetically coupled Co layers $\left(t_{\mathrm{Ru}}=1.2 \mathrm{~nm}\right)$, the angular variation with the external magnetic field is constant $\left(\phi(H)=90^{\circ}\right)$, indicating that the magnetization is always aligned with the external magnetic field. On the other hand, in resonance condition, the antiferromagnetically coupled samples are not completely aligned with the external magnetic field: $H_{R}=1322 \mathrm{Oe}\left(t_{\mathrm{Ru}}=0.7 \mathrm{~nm}\right)$ gives $\phi \sim 26^{\circ}$, while $H_{R}=853 \mathrm{Oe}\left(t_{\mathrm{Ru}}=0.9 \mathrm{~nm}\right)$ gives $\phi \sim 72^{\circ}$. Therefore, the induced rotation of the magnetization caused by the spinflop transition causes a precession at a certain angle that depends on the exchange interaction strength. A precession of the magnetization in an arbitrary direction produces a spin current with a polarization $\sigma$ in that same direction that propagates along the $x$ axis. ${ }^{16}$ It is important to note that in our samples, only one Co layer is in contact with the Pt layer; therefore, the polarization of the spin current is determined by the direction of the magnetization of the Co layer that is in contact with the $\mathrm{Pt}$ film. With the same reasoning, the direction of the charge current as well as the induced electrical field will be determined by the product $\mathrm{J}_{\mathrm{s}}(\hat{x} \times \vec{\sigma})$, where $\hat{x}$ is the direction of propagation and $\vec{\sigma}$ is the polarization direction of the spin current. By performing in-plane FMR measurements together with transversal $\left(V_{\text {ISHE }}^{\mathrm{T}}\right)$ and longitudinal $\left(V_{\text {ISHE }}^{\mathrm{L}}\right)$ ISHE voltage detection, it is possible to measure both longitudinal and transversal ISHE electrical fields caused by the spin-charge current conversion. Figs. 3(a) and 3 (b) present the FMR absorption spectra, $V_{\text {ISHE }}^{\mathrm{T}}$ and $V_{\text {ISHE }}^{\mathrm{L}}$ signals obtained from the SAF-Pt system with 1.2 and $0.7 \mathrm{~nm}$ of $\mathrm{Ru}$ spacer. Conventional $V_{\mathrm{ISHE}}^{\mathrm{T}}$ signals obtained from the ferromagnetically coupled sample $\left(t_{\mathrm{Ru}}=1.2 \mathrm{~nm}\right)$ are similar to SP-ISHE measurements in bilayer systems due to the alignment of the magnetization of the Co layers with $H$ when the resonance condition holds. This also explains the absence of the $V_{\text {ISHE }}^{\mathrm{L}}$ signal.

In contrast, $V_{\mathrm{ISHE}}^{\mathrm{T}}$ and $V_{\mathrm{ISHE}}^{\mathrm{L}}$ signals are observed in the SAF-NM samples $\left(t_{\mathrm{Ru}}=0.7\right.$ and $\left.0.9 \mathrm{~nm}\right)$. For $t_{\mathrm{Ru}}=0.7 \mathrm{~nm}$, the results show a larger amplitude for $V_{\text {ISHE }}^{\mathrm{L}}$ when compared to $V_{\text {ISHE }}^{\mathrm{T}}$, which is explained by the significant misalignment of the magnetization with the external magnetic field. As we have found from the numerical calculation in resonance

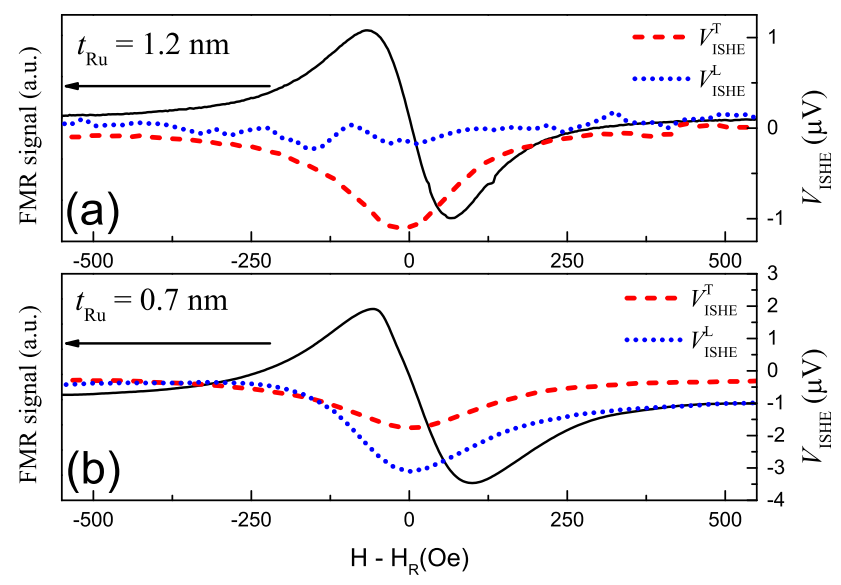

FIG. 3. FMR spectra (continuous line), transversal ( $V_{\text {ISHE }}^{\mathrm{T}}$ ) and longitudinal $\left(V_{\text {ISHE }}^{\mathrm{L}}\right)$ ISHE signals of the system with $t_{\mathrm{Ru}}=1.2 \mathrm{~nm}$ (a) and $0.7 \mathrm{~nm}$ (b). The detection of charge currents via ISHE was performed by using the contact configuration shown in Fig. 1(b).

condition $\left(H_{R} \sim 1322 \mathrm{Oe}\right)$, the magnetization is rotated approximately $64^{\circ}\left(\phi \sim 26^{\circ}\right)$ from the direction of the external magnetic field as a consequence of the large exchange coupling constant $\left(H_{E}=3021 \mathrm{Oe}\right)$. Due to the propagation of the spin current emitted and the induced ISHE signal throughout the $\hat{x} \times \vec{\sigma}$ formula, a $\phi$-dependent $V_{\text {ISHE }}^{\mathrm{T}} / V_{\mathrm{ISHE}}^{\mathrm{L}}$ ratio is expected. An estimation of $V_{\text {ISHE }}^{\mathrm{T}} / V_{\text {ISHE }}^{\mathrm{L}}$ could be obtained from Fig. 2(d). The transversal $\left(E^{T}\right)$ and longitudinal $\left(E^{L}\right)$ components of the induced ISHE electrical field $E_{1}$ as well as $V_{\text {ISHE }}^{\mathrm{T}}$ and $V_{\text {ISHE }}^{\mathrm{L}}$ signals are related through: $V_{\text {ISHE }}^{\mathrm{T}} / V_{\mathrm{ISHE}}^{\mathrm{L}}=\tan \phi$. The ratio calculated from numerical simulations is similar to that extracted from the experimental measurements, within the experimental error: 0.46(5) (calculated) compared to 0.54(5) (experimental). The difference between these values is $\sim 3^{\circ}$ in the angle $\phi$ and can be explained by the presence of a small in plane anisotropy. For the sample $t_{\mathrm{Ru}}=0.9 \mathrm{~nm}$, the ratio $V_{\mathrm{ISHE}}^{\mathrm{T}} / V_{\mathrm{ISHE}}^{\mathrm{L}}=5.3$ (experimental) and $\tan \phi=3.1$ (calculated). The angular difference in this case is around $7^{\circ}$. The relatively large difference found in the $V_{\text {ISHE }}^{\mathrm{T}} / V_{\text {ISHE }}^{\mathrm{L}}$ ratio could be due to the divergence of the tangent function close to $\pi / 2$. Another topic of discussion is related to the generation of the spin current. Our results seem to indicate that the spin current injected into the Pt layer is originated only from the Co layer in contact with the Pt film. In conventional FM-NM systems, a pure spin current is pumped into the $\mathrm{NM}$ as a consequence of an interfacial relaxation process, mediated by the conduction electrons of the metallic layer. ${ }^{16}$ This relaxation mechanism also influences the magnetization dynamics of the FM layer, through the exchange of angular momentum. In this framework, relaxation processes involving the transfer of the angular momentum from the second layer in SAF-NM systems with strong exchange coupling should not be discarded. To further understand the origin of the spin current generation in SAF-NM systems, we measured $V_{\text {ISHE }}^{\mathrm{T}}$ and $V_{\text {ISHE }}^{\mathrm{L}}$ signals after the application of a magnetic field of $\pm 10 \mathrm{kOe}$ along the $y$ axis. Fig. 4 presents the transversal and longitudinal ISHE voltages of SAF-NM structures after being magnetized in opposite directions of the external magnetic field for $t_{\mathrm{Ru}}=0.9 \mathrm{~nm}$ (Figs. 4(a) and 4(b)) and $0.7 \mathrm{~nm}$ (Figs. 4(c) and 4(d)). 


\section{$\mathrm{Co} / \mathrm{Ru}(0.9 \mathrm{~nm}) / \mathrm{Co} / \mathrm{Pt}$}
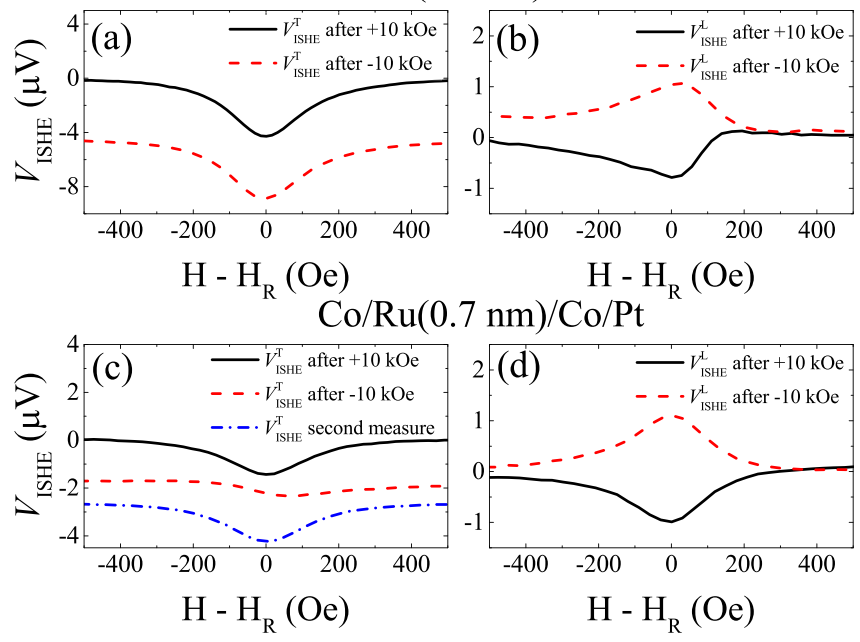

FIG. 4. $V_{\text {ISHE }}^{\mathrm{T}}$ and $V_{\text {ISHE }}^{\mathrm{L}}$ voltages of the $\mathrm{Co} / \mathrm{Ru} / \mathrm{Co} / \mathrm{Pt}$ system after the application of an external magnetic field (10 kOe) along $+y$ and $-y$ axes varying $t_{\mathrm{Ru}}$ : (a) and (b) $0.9 \mathrm{~nm}$ and (c) and (d) $0.7 \mathrm{~nm}$. $V_{\text {ISHE }}^{\mathrm{T}}$ signals in subfigures (a) and (c) have been vertically displaced for a better observation.

The measured $V_{\text {ISHE }}^{\mathrm{T}}$ signal of the sample with $t_{\mathrm{Ru}}=0.9 \mathrm{~nm}$ after the application of $+10 \mathrm{kOe}$ and $-10 \mathrm{kOe}$ behaves similar to the transversal voltages observed in conventional FM-NM bilayers; the obtained signals are independent of the initial state of the magnetization (Fig. 4(a)). However, $V_{\text {ISHE }}^{\mathrm{L}}$ signals in the samples with $t_{\mathrm{Ru}}=0.9 \mathrm{~nm}$ and $t_{\mathrm{Ru}}=0.7 \mathrm{~nm}$ show a sign inversion of the ISHE voltages as observed in Figs. 4(b) and 4(d), respectively. This variation can be explained by taking into account the initial state of the Co layer closer to the Pt film before the detection of the ISHE signal. In order to explain this behavior, we can consider that the Co layers are not perfectly identical, and one of the layers is slightly more anisotropic than the other. In this case, after the application of an external magnetic field of 10 kOe along the $+y(-y)$ direction, the magnetization of the Co layer with a larger anisotropy constant should remain aligned with the magnetic field direction, and due to the AF coupling, the magnetization of the other Co layer should be along the $-y(+y)$ direction. As a consequence, when a magnetic field is applied, the spin-flop transition occurs; the $z$ component of the magnetization of the layer in contact with the Pt film will be different if the saturation field was applied parallel to $+y$ or $-y$ direction. Taking this into account, it is then possible to tune the direction of the magnetization of the Co layer near the Pt electrode before the measurement, thus controlling the sign of the longitudinal ISHE signal. This last result can be interpreted as a mechanism to change the polarization of the spin current emitted during SP experiments. Surprisingly, a reduction of the symmetrical contribution to the transversal ISHE signal was observed after the application of $-10 \mathrm{kOe}$ for the sample with $t_{\mathrm{Ru}}=0.7 \mathrm{~nm}$ of $\mathrm{Ru}$, probably due to a misalignment of the vertical electrical contacts causing undesired contributions. The usual behavior of the transversal signal is recovered in a second measurement as shown in Fig. 4(c).

In conclusion, we have been able to generate pure spin currents using the artificial antiferromagnetically exchanged coupled system $\mathrm{Co} / \mathrm{Ru} / \mathrm{Co}$ with a Pt electrode. Exploiting the magnitude of the exchange coupling and the spin-flop transition in the magnetization process, we have found a way to produce a spin current polarized at different directions. In our case, the spin-flop transition of the SAF/NM system originates a spin current polarized in a direction that depends both on the exchange coupling field and the misalignment of the magnetization. To determine the nature of the produced spin currents "pumped" into the Pt layer, we tuned the magnetization direction of the Co layer near the Pt electrode in different directions. By using a SAF system, we were able to change the polarization of $\mathbf{J}_{\mathbf{s}}$ through the magnetization configuration of the Co layers. We verified that the spin pumping phenomenon is governed by the relaxation process of the ferromagnetic layer in the vicinity of the FM-NM interface. Our results can lead to important advances in hybrid spintronic devices with improved functionalities, particularly the ability to control the polarization direction of spin currents and also the change of the sign of the ISHE signal induced in a metallic layer with the strong spin-orbit interaction.

The authors would like to thank R. Benavides, C. Pérez, and M. Guillén for their extraordinary technical support. This work was partially supported by Conicet (Grant No. PIP 112-201101-00482), ANPCyT (Grant Nos. PICT 20132363 and PICT 2013-0401), and U. N. Cuyo (Grant No. 06/ C421), all from Argentina.

${ }^{1}$ D. Sun, K. J. van Schooten, M. Kavand, H. Malissa, C. Zhang, M. Groesbeck, C. Boehme, and Z. Valy Var-deny, Nat. Mater. 15, 863 (2016).

${ }^{2}$ N. Vlietstra, B. J. van Wees, and F. K. Dejene, Phys. Rev. B 94, 035407 (2016).

${ }^{3}$ M. Wahler, N. Homonnay, T. Richter, A. Mller, C. Eisen- schmidt, B. Fuhrmann, and G. Schmidt, Sci. Rep. 6, 28727 (2016).

${ }^{4}$ J. E. Gómez, J. M. Vargas, L. Avilés-Félix, and A. Butera, Appl. Phys. Lett. 108, 242413 (2016).

${ }^{5}$ K. Ando, S. Takahashi, J. Ieda, Y. Kajiwara, H. Nakayama, T. Yoshino, K. Harii, Y. Fujikawa, M. Matsuo, S. Maekawa, and E. Saitoh, J. Appl. Phys. 109, 103913 (2011).

${ }^{6}$ J.-C. Rojas-Sánchez, N. Reyren, P. Laczkowski, W. Savero, J.-P. Attané, C. Deranlot, M. Jamet, J.-M. George, L. Vila, and H. Jaffrès, Phys. Rev. Lett. 112, 106602 (2014).

${ }^{7}$ O. Mosendz, V. Vlaminck, J. E. Pearson, F. Y. Fradin, G. E. W. Bauer, S. D. Bader, and A. Hoffmann, Phys. Rev. B 82, 214403 (2010).

${ }^{8}$ J. E. Gómez, B. Zerai Tedlla, N. R. Álvarez, G. Alejandro, E. Goovaerts, and A. Butera, Phys. Rev. B 90, 184401 (2014).

${ }^{9}$ C. Hahn, G. de Loubens, O. Klein, M. Viret, V. V. Naletov, and J. Ben Youssef, Phys. Rev. B 87, 174417 (2013).

${ }^{10}$ J. B. S. Mendes, R. O. Cunha, O. A. Santos, P. R. T. Ribeiro, F. L. A. Machado, R. L. Rodríguez-Suárez, A. Azevedo, and S. M. Rezende, Phys. Rev. B 89, 140406 (2014).

${ }^{11}$ H. Wang, C. Du, P. C. Hammel, and F. Yang, Phys. Rev. Lett. 113, 097202 (2014).

${ }^{12}$ H. Saglam, W. Zhang, M. B. Jungfleisch, J. Sklenar, J. E. Pearson, J. B. Ketterson, and A. Hoffmann, Phys. Rev. B 94, 140412 (2016).

${ }^{13}$ Z. Zhang, L. Zhou, P. E. Wigen, and K. Ounadjela, Phys. Rev. B 50, 6094 (1994).

${ }^{14}$ Z. Zhang, L. Zhou, P. E. Wigen, and K. Ounadjela, J. Appl. Phys. 75, 6434 (1994).

${ }^{15}$ J. E. Gómez, M. Guillén, A. Butera, and N. P. Albaugh, Rev. Sci. Instrum. 87, 024705 (2016).

${ }^{16}$ Y. Tserkovnyak, A. Brataas, and G. E. W. Bauer, Phys. Rev. Lett. 88, 117601 (2002). 